Mon. Not. R. Astron. Soc. 000, 000-000 (0000) Printed 15 October $2018 \quad$ (MN LATEX style file v2.2)

\title{
UGC 3672: An unusual merging triplet of gas-rich galaxies in the Lynx-Cancer void
}

\author{
J.N. Chengalur, ${ }^{1 \star}$ S.A. Pustilnik, ${ }^{2} \dagger$ E.S. Egorova ${ }^{3}$ \\ 1 National Center for Radio Astrophysics, TIFR, Pune, India \\ 2 Special Astrophysical Observatory of RAS, Nizhnij Arkhyz, Karachai-Circassia 369167, Russia \\ 3 Sternberg Astronomical Institute, Lomonosov Moscow State University, 13 Universitetsky pr., Moscow, 119991, Russia
}

Accepted October 11, 2016? Received March 3, 2016

\begin{abstract}
We present Hi $21 \mathrm{~cm}$ and optical observations of UGC 3672 which is located near the centre of the nearby Lynx-Cancer void. We find that UGC 3672 consists of an approximately linearly aligned triplet of gas rich dwarfs with large scale velocity continuity along the triplet axis. The faintest component of the triplet is extremely gasrich $\left.\mathrm{M}_{\mathrm{HI}} / L_{\mathrm{B}} \sim 17\right)$ and also extremely metal deficient $(12+\log (O / H) \sim 7.0)$. The metallicity of this dwarf is close to the 'floor' observed in star forming galaxies. Low resolution Hi images show that the galaxy triplet is located inside a common Hi envelope, with fairly regular, disk like kinematics. At high angular resolution however, the gas is found to be confined to several filamentary tidal tails and bridges. The linear alignment of the galaxies, along with the velocity continuity that we observe, is consistent with the galaxies lying along a filament. We argue that the location of this highly unusual system in an extremely low density environment is not a coincidence, but is a consequence of structure formation proceeding more slowly and also probing smaller scales than in regions with average density. Our observations also indicate that wet mergers of galaxies flowing along filaments is a possible pathway for the formation of gas rich disks. The UGC 3672 system provides an interesting opportunity to study the kind of interactions typical between high redshift extremely gas rich unevolved small systems that lie at base of the hierarchical galaxy formation model.
\end{abstract}

Key words: galaxies: evolution - galaxies: dwarf - galaxies: interactions - galaxies: individual: UGC 3672, UGC 3672A - radio lines: galaxies - cosmology: large-scale structure of Universe

\section{INTRODUCTION}

The galaxy distribution in the nearby universe has been known for several decades to contain large regions almost completely devoid of bright galaxies (Jõeveer et al. 1978; Kirshner et al. 1981). Interest in these so called "voids" has been increasing in the recent years for a variety of reasons. They are a prominent feature of the cosmic web. Indeed structure formation in the universe can be regarded as being driven by matter flowing out of the voids and into walls, and then along filaments and finally into the clusters and super-clusters found at the intersection of the filaments. The shape and abundance of voids are sensitive to cosmological parameters (see e.g. Park \& Lee 2007; Biswas et al. 2010) and models of gravity (Cai et al. 2015; Zivick et al. 2015).

\footnotetext{
* E-mail: chengalur@ncra.tifr.res.in (JNC)

$\dagger$ E-mail: sap@sao.ru (SAP)
}

As such comparison of the properties of voids observed in redshift surveys with numerical simulations are an important independent way of constraining the values of dark energy density, (Sutter et al. 2012, e.g.) and possibly even fundamental physics. Further, although voids were first identified as regions devoid of galaxies, numerical simulations as well as observations indicate that voids are not completely empty. The study of galaxies in voids gives one the opportunity to study the effect of environment on the formation and evolution of galaxies.

Numerical simulations predict that the mass function of dark matter halos is strongly influenced by the environment. In void regions its is predicted that the mass function is steeper than in the walls and filaments, i.e. the number of massive halos is depressed compared to that of the low mass halos (e.g. Gottlöber et al. 2003). There is also a spatial segregation, in that the more massive halos are expected to be found nearer the walls which define the void, 
whereas the less massive halos could be found distributed throughout the void (Peebles 2001, e.g.). Observations of void galaxies identified using the SDSS survey do indeed provide observational support for the prediction that the interior of voids are dominated by fainter bluer galaxies (see e.g. Roias et al. 2004; Hovle et al. 2005, 2012; Liu et al. 2015) with higher specific star formation rates (Roias et al. 2005; Moorman et al. 2016) and that there does appear to be a tendency for dwarf galaxies to be dominant as one goes towards the centre of voids (Hovle et al. 2012). Similarly Hi observations of nearby voids find evidence for the HI mass function moving to a lower characteristic mass in the interior of voids (Moorman et al. 2014). Some tension between observation and theory however still remains. For example, the total number of dwarfs found in the interior of voids is significantly smaller than the number of low mass dark matter halos predicted by simulations (Grogin \& Geller 1999; Hoeft et al. 2006).

Although voids are low density regions produced by the streaming of matter out of voids and towards the higher density walls and filaments, (Dubinski et al. 1993; Sheth \& van de Wevgaert 2004, e.g.) they are not devoid of internal structure. Voids contain sub-voids, which are themselves delineated by walls, filaments and high density regions at the intersection of the filaments (e.g. Sahni et al. 1994; Aragon-Calvo \& Szalay 2013). Indeed to first order structure formation in voids is similar to structure formation in a low density universe (Goldberg \& Vogelev 2004). Structure formation in voids proceeds slower than in high density regions, and because the interiors of voids are expanding faster than the average expansion rate, structures in voids are also expanded compared to the mean. Voids hence give one the possibility of looking at both the earlier stages of structure formation as well as probing smaller scales of the power spectrum than is probed by structures in dense regions, and as such, voids can be regarded as "cosmic microscopes and time machines" Aragon-Calvo \& Szalav 2013). Since the effective expansion rate increases towards the central low density regions of the voids these effects get more pronounced the closer one gets to the centre of the void.

For all of the above reasons it is interesting to study galaxies found inside the voids. Recent surveys have found several unusual objects located inside voids. For e.g. Kreckel et al. (2011) discuss the case of KK246, a dwarf galaxy with an extended and disturbed disk lying in the Tully void, and Beygu et al. (2013) discuss the case of an approximately linear triplet of rich galaxies found as part of their Void Galaxy Survey (VGS). They suggest that this linear arrangement may arise due to material flow along a filament inside the void. Numerical simulations of Rieder et al. (2013) provide some support for this suggestion.

We have for some time been conducting a detailed survey of the galaxies in a very nearby void, the Lynx-Cancer void (Pustilnik \& Tepliakova 2011). The Lynx-Cancer void is located at the edge of the Local Volume - its centre only $\sim 18 \mathrm{Mpc}$ distant. Since this is a nearby void, it gives one the opportunity to study galaxies to a much fainter mass and luminosity limit than have been done by mentioned above authors in more distant voids. The void galaxy sample of Pustilnik \& Tepliakova (2011) is estimated to be nearly complete to $\mathrm{M}_{\mathrm{B}}<-14 \mathrm{mag}$, and contains galaxies as faint as $\mathrm{M}_{\mathrm{B}}-11.9 \mathrm{mag}$. The updated version of the sample (in preparation) contains galaxies down to $\mathrm{M}_{\mathrm{B}}-9.6 \mathrm{mag}$. The galaxies in the Lynx-Cancer void tend to be metal deficient compared to the population of a denser environment (Pustilnik et al. 2011, 2016) and the void contains some of the most metal-poor and gas-rich galaxies known (Pustilnik et al. 2005, 2010, 2011; Izotov \& Thuan 2007; Chengalur \& Pustilnik 2013).

A survey of the Hi content of the void galaxies is presented in Pustilnik \& Martin (2016), and HI imaging of a selected subset of gas-rich galaxies is also being undertaken with the GMRT. The ongoing GMRT observations have already led to the discovery of several interesting systems. These include a triplet of extremely gas-rich faint dwarf galaxies J0723+36, once again with an approximately linear alignment (Chengalur \& Pustilnik 2013) and the UGC 4722 system (Chengalur et al. 2015), which is found to consist of an interacting pair of metal-poor galaxies joined by blue plume which appears to consist almost entirely of young stars. One more void triplet is very metal-poor galaxy DDO 68, in which the two more massive components already merged (Ekta et al. 2008), while the third, much fainter component, DDO $68 \mathrm{C}$ at $\sim 42 \mathrm{kpc}$, shows traces of pulled out Hi gas (Cannon et al. 2014). In this paper we discuss UGC 3672 , a galaxy which lies in the central $8 \%$ of the void volume. The galaxy density in the neighbourhood of UGC 3672 has been estimated to be $\sim 10$ times lower than the mean density.

The rest of this paper is arranged as follows. The GMRT observations and the optical photometry are presented in Sec. 2 The results of the observations are presented in Sec. 3 and discussed in Sec. 4] Throughout the paper, we follow Pustilnik \& Tepliakova (2011) in assuming a distance of $16.9 \mathrm{Mpc}$ to UGC 3672 (scale $82 \mathrm{pc} /{ }^{\prime \prime}$ ). The latter distance, in turn, takes into account the large peculiar velocity of $\Delta V \sim-270 \mathrm{~km} \mathrm{~s}^{-1}$ in this region (after Tully et al. (2008)).

\section{OBSERVATIONS AND DATA REDUCTION}

\subsection{GMRT data}

GMRT Hi $21 \mathrm{~cm}$ observations of UGC 3672 were carried out on 8 and 9 September 2015, with a total on source time of $\sim 5.3$ hours. The correlator was configured to a total bandwidth of $4.17 \mathrm{MHz}\left(\sim 890 \mathrm{~km} \mathrm{~s}^{-1}\right)$ divided into 512 channels (or a velocity resolution of $1.74 \mathrm{~km} \mathrm{~s}^{-1}$, the data have not been hanning smoothed since the GMRT FX correlator's spectral leakage is small) centred at the heliocentric redshift of the galaxy. The initial flagging and calibration were carried out using the FLAGCAL pipeline (Prasad \& Chengalur 2012; Chengalur 2013), and the subsequent processing was done using the AIPS package. A continuum image was made using the line free channels and used for self-calibration. The self calibration solutions were then applied to the line visibilities, and the continuum emission subtracted out using the task UVSUB. Images were then made at a variety of resolutions (by applying different UV tapers) using the task IMAGR, and residual continuum subtracted out using the task IMLIN. Images of the integrated Hi emission and the HI velocity field were made using the task MOMNT. 
Table 1. Parameters of the GMRT observations

\begin{tabular}{|c|c|}
\hline & UGC 3672 \\
\hline Date of observations & 2015 Sep 8,9 \\
\hline Field center R.A.(2000) & $07^{h} 06^{m} 27.5^{s}$ \\
\hline Field center Dec. $(2000)$ & $+30^{\circ} 19^{\prime} 19.0^{\prime \prime}$ \\
\hline Central Velocity $\left(\mathrm{km} \mathrm{s}^{-1}\right)$ & 994.0 \\
\hline Time on-source $(\mathrm{h})$ & $\sim 5.3$ \\
\hline Number of channels & 512 \\
\hline Channel separation $\left(\mathrm{km} \mathrm{s}^{-1}\right)$ & $\sim 1.73$ \\
\hline Flux Calibrators & $3 \mathrm{C} 48,3 \mathrm{C} 286$ \\
\hline Phase Calibrators & $0741+312$ \\
\hline
\end{tabular}

\subsection{Photometric data}

Photometry of the UGC 3672 system was done based on the $u, g, r, i$ images taken from the Sloan Digital Sky Survey DR12 (Alam et al. 2015), as well as KPNO $0.9 \mathrm{~m}$ B band and $\mathrm{H}-\alpha$ images from van Zee (2000). For the SDSS releases later than DR8 all imaging data have been recalibrated using the improved procedure described in Padmanabhan et al. (2008). DR12 provides reduced frames after sky-subtraction and this calibration; these can be directly used to derive the photometric fluxes. SDSS fluxes are expressed in a linear scale with units of nanomaggies, from which the Pogson magnitude can be estimated as $m=22.5^{m}-2.5 \log (f)$, where $f$ is the flux expressed in nanomaggies. We convert our integrated $g$ - and $r$ band magnitudes into $B$ band magnitudes $B_{\text {tot }}$ using the transformation formula proposed by Lupton et al. (2005): $B=g+0.313(g-r)+0.2271 ; \sigma=$ 0.0107 . In the case of KPNO $0.9 \mathrm{~m}$ images, the sky subtracted and calibrated frames were downloaded from NED. The $\mathrm{H} \alpha$ and $B$ band magnitudes were computed using the transform formulae provided in the FITS headers.

\section{RESULTS}

\subsection{Low Resolution Data}

We show in Fig. 1 (left) an overlay of the GMRT Hi image (contours; this image is derived from a data cube with a spatial resolution of $58^{\prime \prime} .5 \times 52^{\prime \prime} .6$ and a spectral resolution of $6.8 \mathrm{~km} \mathrm{~s}^{-1}$ ) on a KPNO $0.9 \mathrm{~m} B$ band optical image (greyscales; the image is from van Zee (2000)). The HI emission is spread over a region of $\sim 4.3^{\prime}$ (or $\sim 22 \mathrm{kpc}$ at our assumed distance of $16.9 \mathrm{Mpc}$ ). This is significantly larger than the optical major diameter of $\sim 1.2^{\prime}$. The integrated HI spectrum derived from this datacube is shown in Fig. 2 with the spectrum measured using the Green Bank $43 \mathrm{~m}$ telescope (van Zee et al. 1997; Springob et al. 2005) shown for comparison. The total flux measured at the GMRT is $\sim 10.72 \pm 1.1 \mathrm{Jy} \mathrm{km} \mathrm{s}^{-1}$, where the error bar includes the estimated absolute flux calibration accuracy of $\sim 10 \%$. The flux measured at the GMRT is only $\sim 70 \%$ of the single dish flux. It appears hence that about $30 \%$ of the flux is contained in a diffuse component that is resolved out at the GMRT. A comparison of the two spectra in Fig. 2 indicates that this diffuse emission is fairly uniformly distributed in velocity. It is also worth noting that the spectrum appears to have the standard double horned shape typical of spiral galaxies. The velocity field observed at the GMRT is shown in Fig. 1B].

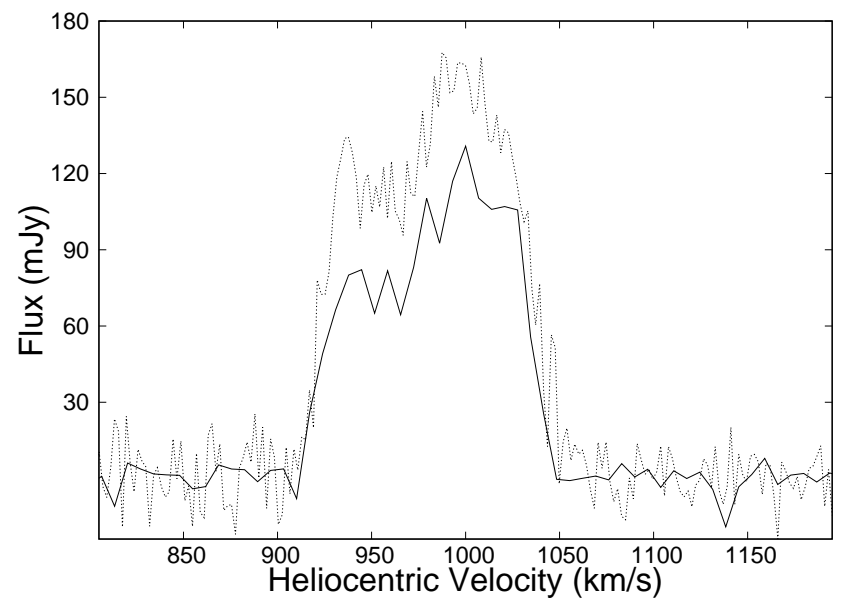

Figure 2. The integrated HI spectrum obtained from the GMRT data cube with spatial resolution of $58^{\prime \prime} .5 \times 52$." 6 and a velocity resolution of $6.8 \mathrm{~km} \mathrm{~s}^{-1}$ (solid line). The dashed line shows the 43-m Green Bank telescope spectrum van Zee et al. 1997; Springob et al. 2005). The GMRT spectrum recovers only about $70 \%$ of the flux detected at $43-\mathrm{m}$ Green Bank telescope. The missing flux, presumably from a diffuse component resolved out at the GMRT is fairly uniformly distributed in velocity.

A smooth gradient can be seen from the north-east to the south-west. The entire system hence appears to be situated inside an Hi envelope with a fairly smooth velocity gradient, consistent with rotation.

The rotation curve for the UGC 3672 system was derived from this low resolution velocity field using a standard tilted ring model, as implemented in the GIPSY 1 , task rotcur. The initial guess for the centre, systemic velocity and position angle were determined using the duchamp package. The initial inclination was taken to be $60^{\circ}$, which is a reasonable estimate from the Hi morphology. These parameters were then determined iteratively using the rotcur package. The final computed position angle and inclination were within a few degrees of the initial guesses, and the final centre also coincides with the initial guess to well within the synthesised beam. The rotation curve fit by rotcur is shown in Fig. 3 The figure shows the rotation curve fit for the approaching and receding half separately (points with error bars) as well as the mean rotation curve determined using both the approaching and receding halves of the galaxy (solid line). The rotation curves derived for the two halves separately are in fairly good agreement, reinforcing the visual impression that, at this spatial resolution the kinematics are fairly regular. The velocity at the last measured point of the rotation curve (which is at a galactocentric radius of $135^{\prime \prime}$, or $11.1 \mathrm{kpc}$ ) is $65 \mathrm{~km} \mathrm{~s}^{-1}$. The corresponding dynamical mass is $\sim 1.0 \times 10^{10} \mathrm{M}_{\odot}$.

\subsection{High Resolution HI images}

Although the low resolution Hi emission appears to be somewhat regular, even from the DSS images of UGC 3672 (not shown) one can see that the galaxy has a peculiar appearance, with a diffuse plume which starts from the northern

1 GIPSY is acronim for Groningen Image Processing SYstem 

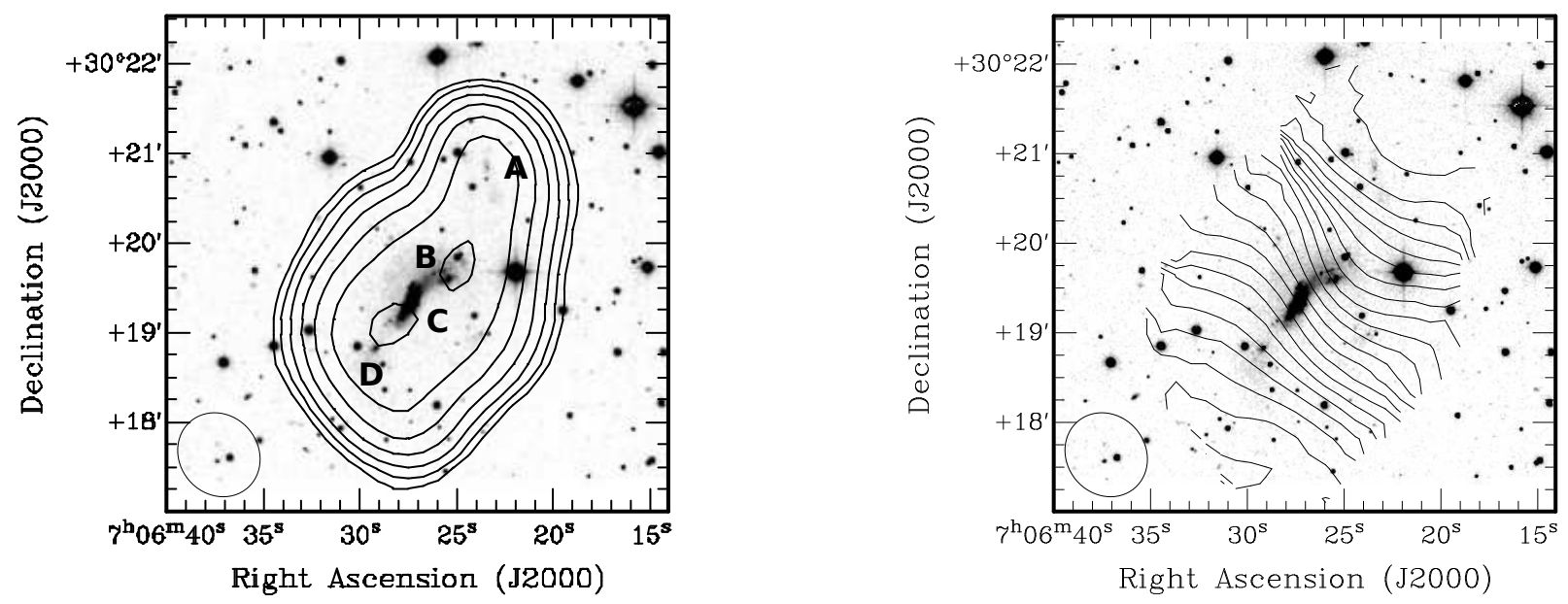

Figure 1. Left Panel: An overlay of the integrated Hi emission (contours) at a resolution of $58^{\prime \prime} .5 \times 52^{\prime \prime} 6$ on a KPNO $0.9 \mathrm{~m} B$ band optical image (greyscales) of the UGC 3672 system. The Hi contours start at a column density of $5.5 \times 10^{19}$ atoms $/ \mathrm{cm}^{-2}$ and are spaced a factor of 1.5 apart. See the text for the labelling of the different components. The beam is shown in the bottom left. Right Panel: Velocity field obtained from the Hi data cube (the first moment map). The spatial resolution is $58^{\prime \prime} 5 \times 522^{\prime \prime} 6$. The velocity contours go from $929 \mathrm{~km} \mathrm{~s}^{-1}$ to $1040 \mathrm{~km} \mathrm{~s}^{-1}$ in steps of $5 \mathrm{~km} \mathrm{~s}^{-1}$. A smooth velocity gradient, consistent with that expected from rotation is seen across the entire system.

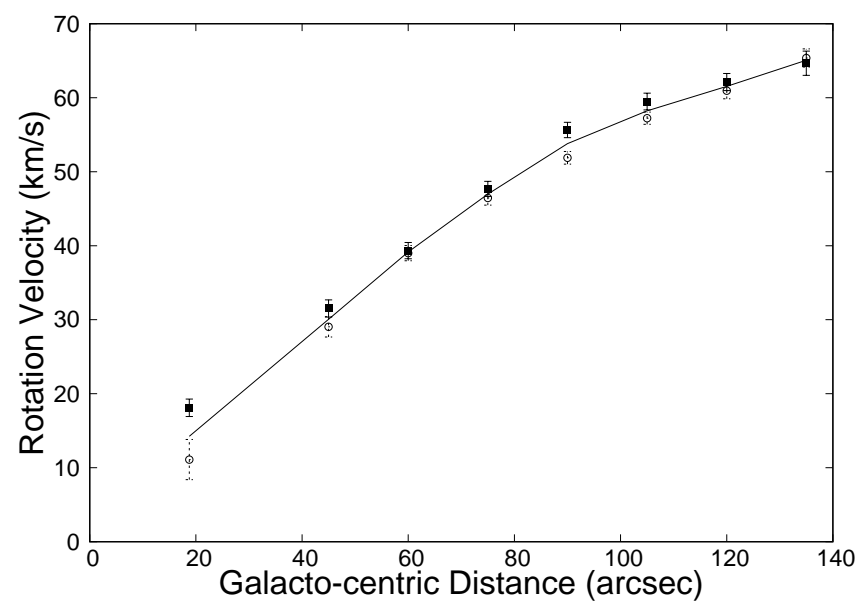

Figure 3. The rotation curve for UGC 3672, derived using the GIPSY program rotcur from the velocity field at a resolution of $588^{\prime \prime} 5 \times 52$ "' 6 . The points with the error bars show the rotation curve as derived for the approaching and receding sides separately, while the solid line shows the rotation curve derived using the entire galaxy. There is reasonably good agreement between the rotation curves derived from the two halves separately. end of the brightest emission and extending westwards. This feature can also be seen in the KPNO $0.9 \mathrm{~m} B$-band image (Fig. 1). In the $B$-band image one can also see a knot of emission to the south-east of the main body and faint diffuse emission about $1^{\prime}$ to the north-west. The KPNO $0.9 \mathrm{~m} \mathrm{H} \alpha$ image of the galaxy (van Zee 2000) also shows a faint knot of emission coincident with the diffuse emission to the north-west seen in the $B$ band image. van Zee (2000) does not comment about this $\mathrm{H} \alpha$ knot, but it can be seen clearly in Fig. 4 of the current paper. This emission has also been identified as arising from a companion dwarf galaxy by Pustilnik et al. (2016) based on the spectra obtained with the SAO 6m telescope. As discussed further below, our $\mathrm{HI}$ data also supports this conclusion. We henceforth refer to this galaxy as component A or UGC 3672A. The peculiar morphology of UGC 3672 along the presence of a nearby companion galaxy that is embeded in a common Hi envelope makes it interesting to look at the optical and Hi images in more detail.

To start with we note that the optical $B$ band image for UGC 3672 itself is peculiar, consisting of a diffuse low surface brightness structure which we henceforth call component B, (see the labels in Fig. 1) attached to the north west of a relatively high surface brightness narrow emission region (which we henceforth refer to as component $\mathrm{C}$ ). The 


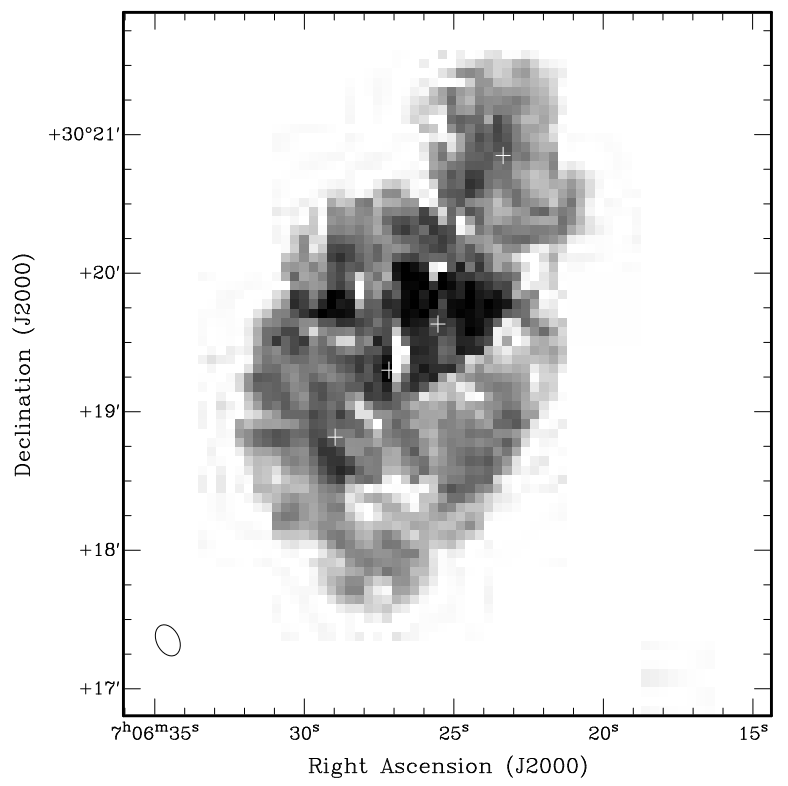

Figure 5. Greyscale representation of the second moment of the Hi emission at a resolution of $14^{\prime \prime} 6 \times 10^{\prime \prime} 1$. The beam size is shown in the lower left corner. The locations of the components A to D (going from north to south) are marked by crosses. All four components are associated with local peaks in the velocity dispersion.

$\mathrm{H} \alpha$ image (Fig. 4) shows knots of bright $\mathrm{H} \alpha$ emission associated with both of these structures, as well as a faint knot of $\mathrm{H} \alpha$ emission south-east of the main galaxy (which we henceforth refer to as component D). In addition, as discussed above, there is also $\mathrm{H} \alpha$ emission associated with the companion galaxy UGC 3672A. The Hi emission can also be seen have a filamentary structure (see Fig. 4). As discussed in Sec. 4.1 these filaments can be understood as tidal tails arising from the interaction of extremely gas rich progenitors. We also show in Fig. 5 the second moment (velocity dispersion) of the Hi distribution. An increase in the velocity dispersion near the location of all the four optical components $(\mathrm{A}, \mathrm{B}, \mathrm{C}, \mathrm{D})$ can be seen.

\subsection{Magnitudes, colours and $\mathrm{H} \alpha$ fluxes}

In Table 2 we present the results of the photometry for components A, B and C+D. Following Schlafly \& Finkbeiner (2011) we take the $B$ band extinction to be $A_{\mathrm{B}}=0.26^{m}$. This value was used for computing both the absolute magnitudes as well as the colour corrections. In Table 2 we show $g_{\text {tot }}$ measured from the SDSS images as well as the derived $B$ magnitudes. We also show the $(u-g),(g-r)$ and $(r-i)$ colours for the different components. For deriving the colours we only use regions with a detectable signal in the $u$ band. The $B$ band magnitudes were however estimated over larger apertures using the higher signal to noise ratio $g$ and $r$ band images. For components $\mathrm{B}$ and $\mathrm{C}$, the measured colours are the same within the uncertainties, we hence tabulate the colours derived for the sum of these components.

We also measured the the $B$-band and $\mathrm{H} \alpha$ magnitudes of the different components using the KPNO $0.9 \mathrm{~m}$ images from van Zee (2000). The total $B$ magnitude for sum of B, C
Table 2. Photometric parameters of UGC 3672 system

\begin{tabular}{lccc}
\hline Property & Comp. A & Comp. B & Comp. C+D \\
\hline$B_{\text {tot }}(\mathrm{SDSS})$ & $18.93 \pm 0.15$ & $17.09 \pm 0.08$ & $16.32 \pm 0.04$ \\
$B_{\text {tot }}(\mathrm{KPNO})$ & 19.09 & 17.15 & 16.26 \\
$M_{\mathrm{B}}^{1}(\mathrm{mag})$ & -12.47 & -14.31 & -15.08 \\
$F_{\mathrm{H} \alpha}^{2}$ & $26 \pm 4.8$ & $139 \pm 6.6$ & $296 \pm 9.4$ \\
$\mu_{\mathrm{B}}^{3}$ & 25.27 & $\ldots$ & $\ldots$ \\
$g_{\text {tot }}$ & $19.39 \pm 0.13$ & $17.24 \pm 0.05$ & $16.20 \pm 0.03$ \\
$(u-g)_{\text {tot }}$ & $0.81 \pm 0.33$ & \multicolumn{2}{c}{$0.86 \pm 0.07$} \\
$(g-r)_{\text {tot }}$ & $-0.08 \pm 0.18$ & \multicolumn{2}{c}{$0.31 \pm 0.04$} \\
$(r-i)_{\text {tot }}$ & $-0.29 \pm 0.18$ & \multicolumn{2}{c}{$0.16 \pm 0.03$} \\
$(u-g)_{\text {outer }}$ & $\ldots$ & \multicolumn{2}{c}{$0.92 \pm 0.11$} \\
$(g-r)_{\text {outer }}$ & $\ldots$ & \multicolumn{2}{c}{$0.23 \pm 0.06$} \\
$(r-i)_{\text {outer }}$ & $\ldots$ & \multicolumn{2}{c}{$0.12 \pm 0.05$} \\
\hline
\end{tabular}

1. Adopted from the SDSS derived $B_{\text {tot }}$ and the distance module of $31.14^{m}$, to obtain the robust value of $M(\mathrm{HI}) / L_{\mathrm{B}}$. Corrected for galactic extinction $A_{\mathrm{B}}=0.26^{m}$. 2. $\mathrm{H} \alpha$ in units of $10^{-16} \mathrm{ergs} \mathrm{cm}^{-2} \mathrm{~s}^{-1}$. 3. mag $\operatorname{arcsec}^{-2}$, average $B$-band surface brightness within the isophote of $\mathrm{SB}(\mathrm{B})=26.54^{m} \operatorname{arcsec}^{-2}$, corrected for $A_{\mathrm{B}}=0.26^{m}$, but not for inclination. The latter dims the SB by additional $(1.3-1.8)^{m} \operatorname{arcsec}^{-2}$ for the intrinsic disc axial ratios of $q=0.2-0.3$. Total magnitudes, $F_{\mathrm{H} \alpha}$ and all colours are not corrected for galactic extinction. 4. the light of components $\mathrm{C}$ and D is summed up for $B_{\text {tot }}$ and $M_{\mathrm{B}}$ and for parameter $M(\mathrm{HI}) / L_{\mathrm{B}}$ since for low resolution Hi map, used for the estimate of HI-fluxes they are situated in a common HI cloud.

and D components estimated by us is somewhat fainter than that derived for the whole UGC 3672 system by van Zee (2000). This is likely due to the difference in the sizes of apertures used. Our $B$ magnitude for the sum of B and $\mathrm{C}+\mathrm{D}$ components is however in accord with UGC 3672 total magnitude from Perepelitsyna et al. (2014) and also with that derived from our SDSS $g, r$ magnitudes.

A zoom in on component A is shown in Fig [7 One can see that the Hi emission peaks near the star forming region, and that in addition to the star forming knot, there is also a bright foreground star projected on to the diffuse low surface brightness optical emission. The foreground star was masked out in order to compute the total magnitude. As before for deriving the colours we only use regions with a detectable signal in the $u$ band. The $B$ band magnitudes were however estimated over larger apertures using the higher signal to noise ratio $g$ and $r$ band images.

\section{DISCUSSION}

\subsection{The nature of UGC 3672}

The low resolution HI images suggest that we are dealing with a somewhat clumpy rotating gas disk, a not unusual situation for dwarf galaxies (see e.g. Begum et al. 2008). The optical images however show that the system appears to be disturbed, and as we discussed above, the Hi data indicate that UGC 3672 is undergoing an interaction with a much fainter gas rich companion galaxy which we have named UGC 3672A.

We now look at the UGC 3672 system as a whole. We show in Fig. 4 a greyscale representation of the integrated 

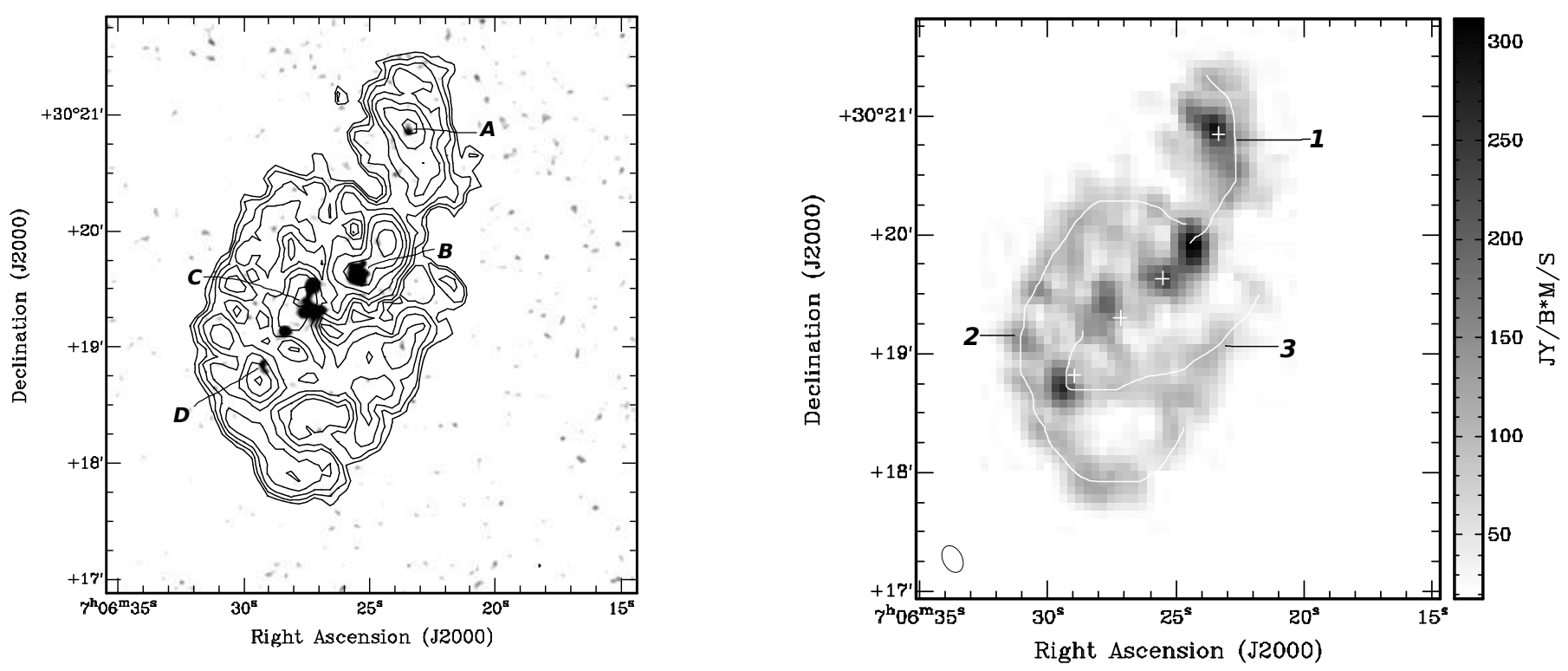

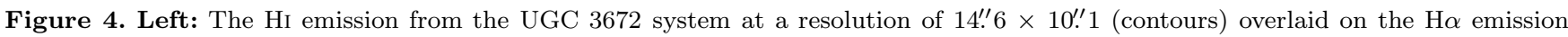
(greyscales). The H $\alpha$ data is from van Zee (2000), and has been smoothed to a resolution of $3^{\prime \prime}$. The Hi contours start at $1.7 \times$ $10^{20}$ atoms $/ \mathrm{cm}^{-2}$ and increase in steps of $\sqrt{2}$. Right: Greyscale representation of the Hi emission at a resolution of $14^{\prime \prime} 6 \times 10^{\prime \prime} 1$. The beam size is shown in the lower left corner. The locations of the components A to D (going from north to south) are marked by crosses. Three filamentary structures $(1,2,3)$ are also marked. As discussed in the text, the velocity varies continuously along these filaments.

Hi at $\sim 14^{\prime \prime}$ resolution. The locations of the components $\mathrm{A}, \mathrm{B}, \mathrm{C}$ and $\mathrm{D}$ are also shown using crosses (with A being the northernmost and D the southernmost). Peaks in the HI emission are seen near all of the components, although the Hi peak near the brightest optical component (C) is comparatively less bright. The filamentary structure of the HI distribution can also be seen. To guide the eye and also to correlate this image with the channel maps in Fig. 6, three filaments labelled 1,2,3 have also been demarcated. The continuity of the velocity fields along these three filaments can be seen in Fig. 6. The centres of the four components A,B,C and $\mathrm{D}$ are once again marked with crosses. At the highest velocity channel $(1024.3 \mathrm{~km} / \mathrm{s})$ the south-west extreme of filament 3 can be seen. In the lower velocity channels one can see that the emission from this filaments moves towards the north-east, passes through component D and then continues to the east of the main optical emission (i.e. the line joining the central two crosses) and finally terminates (at a velocity $\sim 975.8 \mathrm{~km} / \mathrm{s}$ ) slightly to the north of component B. Emission from filament 2 starts a little south of component $\mathrm{C}$ (at a velocity $\sim 1003.5 \mathrm{~km} / \mathrm{s}$ ) and it too overlaps with region $\mathrm{D}$, before continuing to the north-west of the main optical emission and terminating (at velocities $\sim 962 \mathrm{~km} / \mathrm{s}$ ). Filament 1 can be seen starting from the north of component B (at velocities $\sim 948.2 \mathrm{~km} / \mathrm{s}$ ) and continues northward until it terminates near component A (at velocities $\sim 920.5 \mathrm{~km} / \mathrm{s}$ ).

In view of all of this, we propose the following model for this system. UGC 3672 itself represents the merger of two progenitors, viz. the components $\mathrm{B}$ and $\mathrm{C}$. Tidal interaction between these, as well as the third galaxy UGC 3672A has resulted in the filamentary structures, which are essen- tially tidal tails and arms. As we saw above, the star forming component D is associated with the intersection of two of these tidal tails. A possible alternate scenario is one where there are only two galaxies, viz. UGC 3672 and the companion UGC $3672 \mathrm{~A}$. In such a scenario one would require that the material that forms component $\mathrm{B}$ has been tidally stripped from the main body of UGC 3672 by the companion UGC 3672A. This seems an unlikely scenario given that UGC 3672A is significantly less massive than UGC 3672. Support for our conjecture that component B was originally a distinct system is also provided by the $\mathrm{H} \alpha$ kinematics from Fabri-Perot scanning interferometer observations by Moiseev (2014). Moiseev (2014) notes that the kinematics of the system is highly disturbed, with the kinematical position angle changing by almost $90^{\circ}$ as one moves from the centre outwards (more or less coincident with going from radii that correspond to component $\mathrm{C}$ to radii including components $\mathrm{C}$ and B). Based on the $\mathrm{H} \alpha$ kinematics Moiseev (2014) speculate that this is possibly because this object is a merger remnant, in line with our suggestion that $\mathrm{C}$ and $\mathrm{B}$ were once distinct entities.

We have so far been treating component A as a separate galaxy. Is there any kinematic evidence for this? We show the velocity field as well as the second moment map of the Hi emission around U 3672A in Fig. 7 As can be seen the velocity gradient (which corresponds to that along filament 1 in Fig. 4) starts out in the north-south direction, but near component $\mathrm{A}$ it the gradient changes direction and is aligned along the east-west direction. The second moment map Fig. 7 (right panel) also shows an increase in the velocity dispersion around component A. As such, component A, 


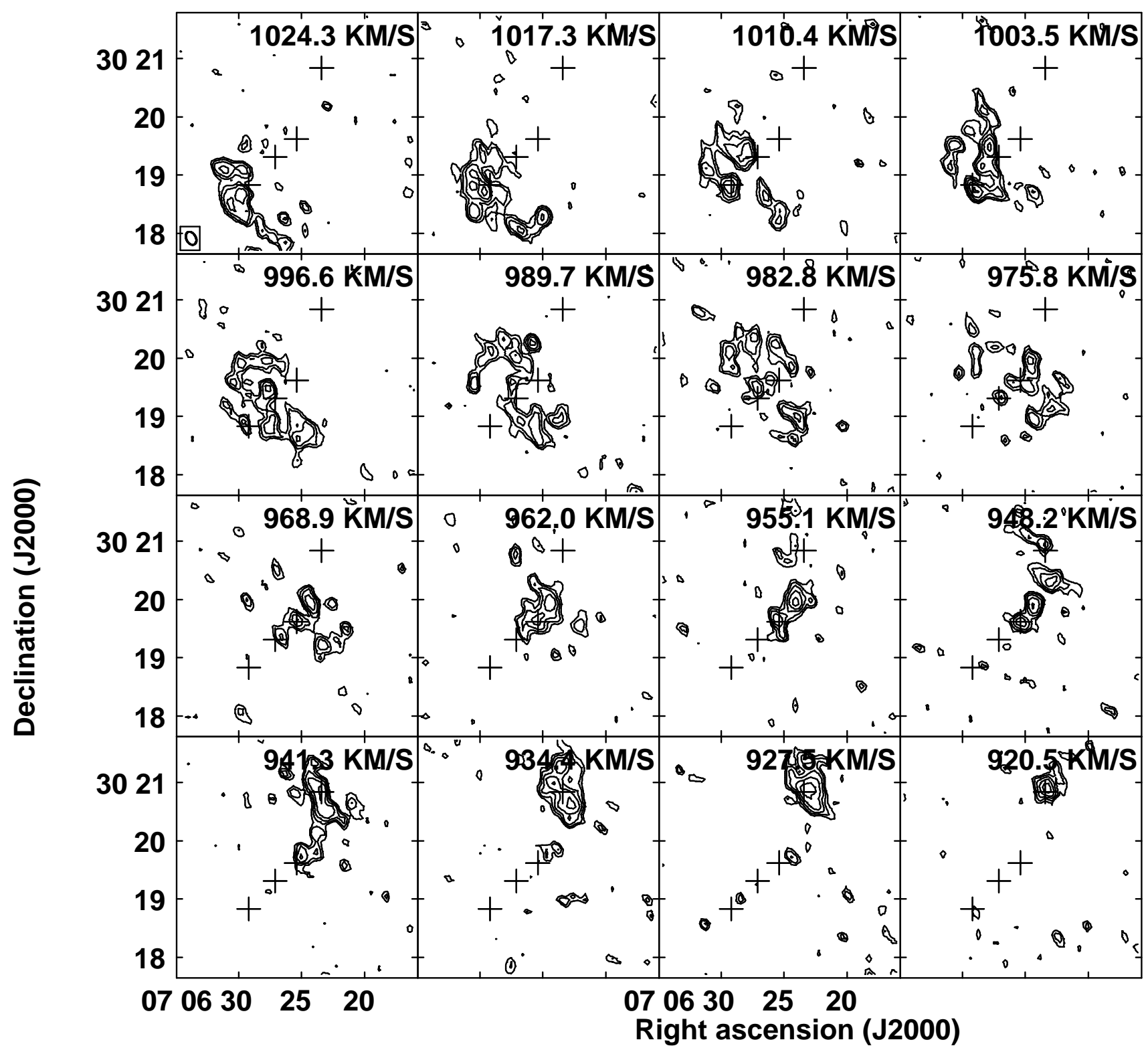

Figure 6. Channel maps of the Hi emission from the U3672 at 14 " $\times 10^{\prime \prime}$. These channels do not cover most of the emission, and this velocity range was selected to clearly bring out the velocity continuity along the filaments marked in Fig. 4 The contours are logarithmically equally spaced between $2 \mathrm{mJy} / \mathrm{Bm}$ and $8 \mathrm{mJy} / \mathrm{Bm}$. The channel width is $\sim 6.9 \mathrm{~km} / \mathrm{s}$. The crosses mark the centres of the components A,B,C,D going from north to south. The beam size is shown in the lower left corner of the first panel.

appears to be a kinematically distinct component, consistent with our treatment of it as a separate galaxy.

Assuming then that the UGC 3672 system represents the merger of what was originally a galaxy triplet, it is interesting to determine the properties of the progenitor systems. We list in Table 3 the $B$ band magnitudes and luminosities as well as the Hi fluxes and masses of the progenitors. We have measured the fluxes using the AIPS task BLSUM, summing over the regions around each optical component.
We note that the identification of the Hi associated with the different components is somewhat subjective, given the complex nature of the HI distribution. The measurements given below are hence somewhat uncertain. The uncertainty is probably least for component A, given that it is reasonably well separated from the rest of the emission.

Summarizing the properties of the triplet components in Table 3, it is worth emphasizing the extremely large ratio $M(\mathrm{HI}) / L_{\mathrm{B}}=17.1$ (in solar units) for the faint companion 

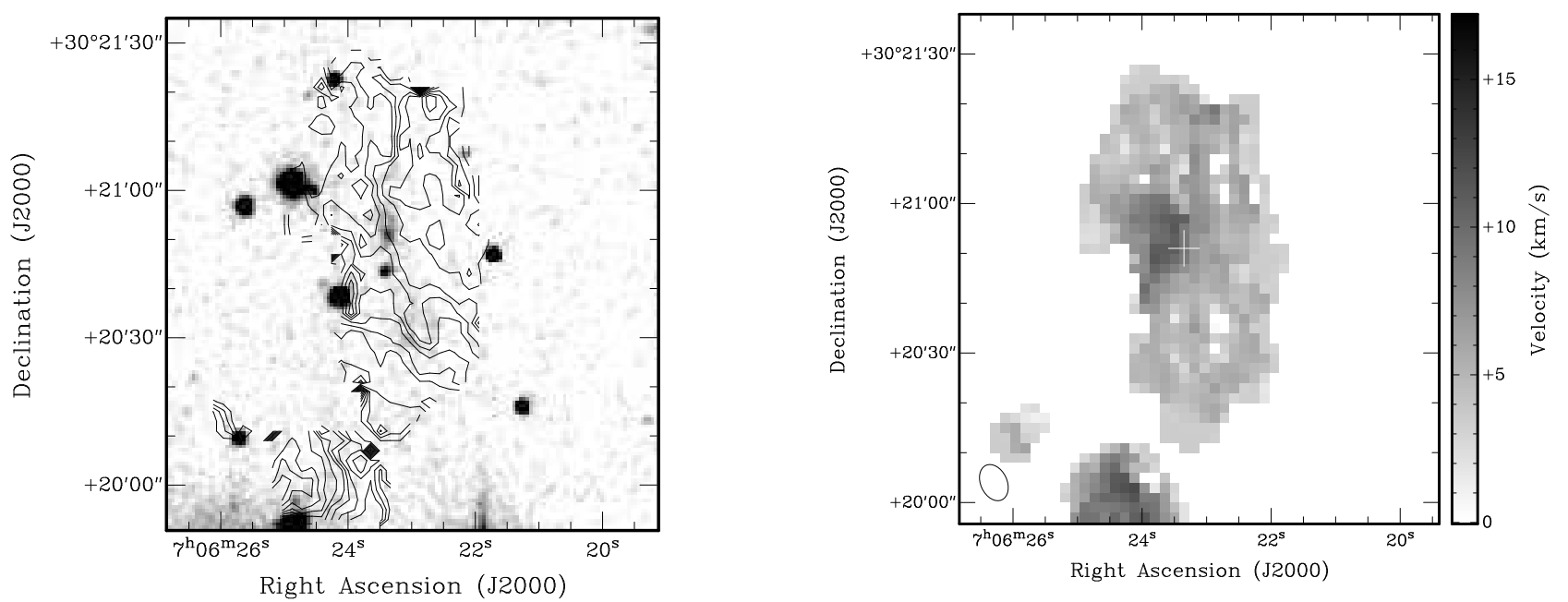

Figure 7. left An overlay of the Hi velocity field at $8 .{ }^{\prime \prime} 1 \times 55^{\prime \prime} 5$ (contours) on the KPNO $0.9 \mathrm{~m} B$-band image of UGC $3672 \mathrm{~A}$. The overall North-South extent of the very low surface brightness optical emission is $\sim 40^{\prime \prime}$. A faint foreground star is also seen superposed on the diffuse optical emission. A change in the direction of the gradient of the Hi velocity field around U3672A can also be seen. right An greyscale representation of the second moment of the Hi distribution at 8 .' $1 \times 5$." 5 . The beam size is shown in the lower left corner. An increase in the velocity dispersion around U3672A can be seen.

Table 3. Properties of the progenitors in system UGC 3672

\begin{tabular}{|c|c|c|c|}
\hline Property & Comp. A & Comp. B & Comp. C $+\mathrm{D}$ \\
\hline$B_{\text {tot }}^{1}(\mathrm{mag})$ & 18.93 & 17.09 & 16.32 \\
\hline$M_{\mathrm{B}}^{2}(\mathrm{mag})$ & -12.47 & -14.31 & -15.08 \\
\hline$L_{\mathrm{B}}^{2}\left(10^{7} L_{\odot}\right)$ & 1.51 & 8.24 & 16.7 \\
\hline$V_{\text {hel }}^{3}\left(\mathrm{~km} \mathrm{~s}^{-1}\right)$ & $936.7 \pm 1.3$ & $976.5 \pm 1.3$ & $1015.9 \pm 3.5$ \\
\hline $\mathrm{S}_{\mathrm{HI}}\left(\mathrm{Jy} \mathrm{km} \mathrm{s}^{-1}\right)$ & $2.38 \pm 0.5$ & $3.66 \pm 0.7$ & $4.7 \pm 0.8$ \\
\hline $\mathrm{S}_{\mathrm{HI}}^{4}\left(\mathrm{Jy} \mathrm{km} \mathrm{s}^{-1}\right)$ & $3.41 \pm 0.7$ & $5.21 \pm 1.0$ & $6.73 \pm 1.1$ \\
\hline $\mathrm{M} 1_{\mathrm{HI}}^{5}\left(10^{7} M_{\odot}\right)$ & $25.8 \pm 5$ & $35 \pm 7$ & $45 \pm 8$ \\
\hline $\mathrm{M} 1_{\mathrm{HI}} / L_{\mathrm{B}}\left(M_{\odot} / L_{\odot}\right)$ & 17.1 & 4.2 & 2.7 \\
\hline$L_{\mathrm{H} \alpha}\left(10^{37} \mathrm{ergs} \mathrm{s}^{-1}\right)$ & 9.8 & 52.3 & 124.0 \\
\hline $\operatorname{SFR}^{6}\left(M_{\odot} / \mathrm{yr}\right)$ & 0.0006 & 0.0031 & 0.0074 \\
\hline
\end{tabular}

1. As obtained from the SDSS images. 2. For the assumed here and below distance of $16.9 \mathrm{Mpc}$, and including a galactic extinction of $A_{\mathrm{B}}=0.26^{\mathrm{m}}$ (Schlafly \& Finkbeiner 2011). 3. Taken from the $\sim 60^{\prime \prime}$ resolution data cube. The uncertainties in this and all of the Hi quantities listed here include also those related to assigning emission to a given component. 4. This is derived from the above row, but assuming that the total flux is as measured at 43-m Green Bank telescope (43mGB), and that the flux in each component is scaled proportionately. 5. Derived from the fluxes scaled to the $43 \mathrm{mGB}$ value. 6. Star Formation Rate as derived from $\mathrm{H} \alpha$ luminosity $L_{\mathrm{H} \alpha}$ with the formula from Hunter \& Elmegreen (2004).

LSBD UGC 3672A. This object is one of a handful of galaxies with very high, well measured $M(\mathrm{HI}) / L_{\mathrm{B}}$ ratios. We note also that two other very gas-rich LSBDs have been found in the central regions of the Lynx-Cancer void. The others two being J0723+3622 and J0723+3624 with $M(\mathrm{HI}) / L_{\mathrm{B}} \sim 10$ and 26, respectively (Chengalur \& Pustilnik 2013). Components 'B' and ' $\mathrm{C}+\mathrm{D}$ ', are also gas rich having $M(\mathrm{HI}) / L_{\mathrm{B}}$ of 4.2 and $\sim 2.6$, respectively. The total $B=15.87^{m}$ that we measure is $\sim 0.44 \pm 0.20^{m}$ however fainter than that given by van Zee (2000) for UGC 3672. As discussed above we believe that our values are the appropriate ones. If however we adopt the van Zee (2000) measurement of $B$ (UGC3672) $=$ $15.43^{m}$, then the parameter $M(\mathrm{HI}) / L_{\mathrm{B}}$ for the sum light of components ' $\mathrm{B}$ ' and ' $\mathrm{C}+\mathrm{D}$ ' drops from $\sim 3.2$ to $\sim 2.1$.

We also use the observed colours and PEGASE2 models with continuous as well as starburst models to try and constrain the ages of the different components. In Fig. 8 we show in plot $(g-r)$ vs $(u-g)$ the positions of all UGC 3672 system components, with the overlaid PEGASE2 evolutionary tracks. For the left panel, the adopted value of stellar metallicity $z=0.004$ is close to that measured for HII regions in components $\mathrm{B}$ and $\mathrm{C}(12+\log (\mathrm{O} / \mathrm{H}) \sim 8.0)$. For the very gas-rich and low-mass component $\mathrm{A}$, the measured recently value of $12+\log (\mathrm{O} / \mathrm{H}) \sim 7.0$ (Pustilnik et al. 2016), corresponds to a metallicity of $z=0.0004$ which is adopted for the evolutionary tracks at the right panel.

Similar to several other faint LSB dwarfs found among the Lynx-Cancer galaxies by Perepelitsyna et al. (2014), component A shows very blue ugr colours. This indicates a small age for its main stellar population, although we note that the uncertainties are large. Specifically, for component $\mathrm{A}$, the age range for the nearest model track corresponds to 
the instantaneous starburst model with elapsed time from the burst of $\sim 0.1$ Gyr. The track for continuous SF in this region of $u g r$ plot runs quite close to instantaneous track, and the corresponding age ranges are $T \sim 0.3-1$ Gyr, depending on the assumed form of IMF, Salpeter or Kroupa, respectively. We note also that the age estimates for component $\mathrm{A}$ are much smaller than those for the outer parts of components B and C.

\subsection{UGC 3672 in context}

As mentioned in the introduction, UGC 3672 lies in the central $8 \%$ of the Lynx-Cancer void volume. For the Lynx-Cancer void as a whole, the density of galaxies with $M_{\mathrm{B}} \lesssim-14$ is estimated to be $\sim 10 \%$ of the mean value (Chengalur \& Pustilnik 2013). UGC 3672 is also a member of the Catalog of Nearby Isolated galaxies (Karachentsev et al. 2011). UGC 3672 is clearly located in a highly unusual region. As described above, the properties of the system are also highly unusual. It appears unlikely that the fact that the system is highly unusual as well as its environment is higly unusual is just a coincidence; it appears more reasonable to conjecture that finding such a system near the center of a void is related to the "cosmic microscope and time machine" effects associated with the lowest density regions of the universe. An additional evidence for this effect is the finding of another unusual system - very gas-rich dwarf triplet J0723+36, also near the void centre, only at $\sim 2.2 \mathrm{Mpc}$ from UGC 3672 (Chengalur \& Pustilnik 2013).

In this context, it is interesting to note that the UGC 3672 system in some ways resembles a scaled down version of well known nearby interacting groups, e.g., the M81 group (Yun et al. 1994). In the M81 group also one can see tidal streamers and bridges connecting the galaxies in the group. The total HI mass of the M81 system is however $\sim 5 \times 10^{9} M_{\odot}, \sim 5$ times larger than the total HI mass of the UGC 3672 system. The length scales are also similiarly a factor of $\sim 7$ larger, with the total extent of the M82 system being $\sim 150 \mathrm{kpc}$, and the individual tidal streamers being $\sim 60 \mathrm{kpc}$ in length.

It is also interesting to note the ongoing star formation in the tidal features in UGC 3672, particularly the bright star forming knot in component 'D' where two separate streamers appear to intersect. This is similar to what is seen in other larger tidal tails (e.g. Hibbard et al. 2005; Neff et al. 2005).

Star formation in the companion galaxy UGC 3672A is also interesting. Numerical simulations of star formation triggered by tidal interactions of gas-rich dwarf systems show that this can lead to bright, compact central star forming regions, similar to what we see here (Bekki 2015b). Such interactions could also lead to the formation of extremely metal defficient (XMD) galaxies (Ekta \& Chengalur 2010; Bekki 2008, 2015a). Indeed, the fresh 6-m SAO telescope spectroscopy of the faint HII region in UGC 3672A reveals an extremely low gas metallicity $\left(\mathrm{O} / \mathrm{H} \sim(\mathrm{O} / \mathrm{H})_{\odot} / 50\right.$; Pustilnik et al. 2016, submitted). In an alternative scenario, based on the cold ambient intergalactic gas accretion along the dark matter filaments (e.g. Sanchez Almeida et al. 2014, 2015, and references therein), one expects an asymmetric location of starburst (similar to tadpole galaxies), with the lo- cally reduced metallicity being due to the mixture of galaxy gas with the ambient accreted intergalactic gas.

The metallicity of UGC 3672A is extremely low, and is in fact close to the metallicity floor $\sim Z_{\odot} / 50$ found for both the star forming regions of XMDs and BCGs as well as their outer envelopes (Thuan et al. 2005; Izotov et al. 2012; Guseva et al. 2015, and references therein). The presence of this 'floor' is postulated to reflect pre-enrichment by Population III stars of the material out of which these galaxies formed. This makes it interesting to speculate whether UGC 3672A has undergone most or all of its star formation fairly recently, i.e. timescales $\left(T_{\mathrm{SF}} \lesssim 1 \mathrm{Gyr}\right.$ as its $(u-g)$, $(g-r)$ colours in Fig. 8 indicate). Such a very late 'recent' onset of SF can be related to the inflow along the void filament to the pre-existing pair of currently merging components ' $\mathrm{B}$ ' and 'C'. The tidal action of the 'massive' DM halo of this pair could induce disturbance and related star formation on timescales of $\lesssim 1$ Gyr. The crucial tests for checks of various alternatives scenarios for star formation in UGC 3672A would require studies of the resolved old stellar population with the next generation giant optical telescopes like E-ELT, TMT and JWST.

Recently (Hirschauer et al. 2016) reported the discovery of another record-low metallicity dwarf SDSS J094332.35+332657.6 identified with the ALFALFA HI-source AGC 198691 Its metallicity $(12+\log (\mathrm{O} / \mathrm{H})=7.02 \pm 0.03$ derived via the direct method $)$, is close to the mentioned above 'floor'. This faint $\left(B_{0}=19.76\right)$ extremely gas-rich dwarf with $M(\mathrm{HI}) / L_{\mathrm{B}}=6.5$ is also very blue and probably unevolved. The galaxy is situated at an angular distance of $\sim 13^{\prime}$ from the $3.5^{m}$ brighter

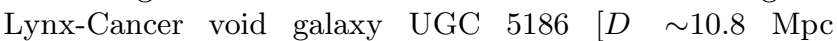
(Pustilnik \& Tepliakova 2011)]. The heliocentric velocity of AGC 198691 is $V_{\text {hel }}=514 \mathrm{~km} \mathrm{~s}^{-1}$, only $35 \mathrm{~km} \mathrm{~s}^{-1}$ smaller than its brighter counterpart. If we assume that these two galaxies form a bound system, their angular separation corresponds to a linear projected separation of $\sim 40 \mathrm{kpc}$. Then one could expect to see signs of tidal disturbance in the morphology and kinematics of the smaller galaxy in deep Hi observations. If AGC 198691 is not bound to UGC 5186, it may reside $\sim 0.5 \mathrm{Mpc}$ closer and belong to one of the other filaments in the galaxy distribution identified in Pustilnik et al. (in preparation). Thus, this new record-low metallicity object may have a nature similar to that of UGC3672A, emphasising once more the potential of finding atypical low-mass galaxies in voids.

In contrast to UGC $3672 \mathrm{~A}$, star formation is much more wide spread in the remainder of the system. Metallicity information is available for two HiI regions in the system (van Zee et al. 1997), one each in what we have designated here as components ' $\mathrm{B}$ ' and 'C'. Both measurements agree within the error bars and the average oxygen abundance is $12+\log (\mathrm{O} / \mathrm{H})=8.01 \pm 0.1$. This value is very close to $7.99 \pm 0.05$, obtained on the independent determination in Pustilnik et al. (2011). Both values of $\mathrm{O} / \mathrm{H}$ are consistent with what is expected from the luminosity-metallicity relation (Ekta \& Chengalur 2010; Berg et al. 2012).

In the context of its location deep inside a void, the well defined tidal tails and bridges in the UGC 3672 system, as well as the fact that the diffuse gas looks like it is settling into a rotating disk, is interesting. The role of mergers in determining the morphology of current day galaxies has long 

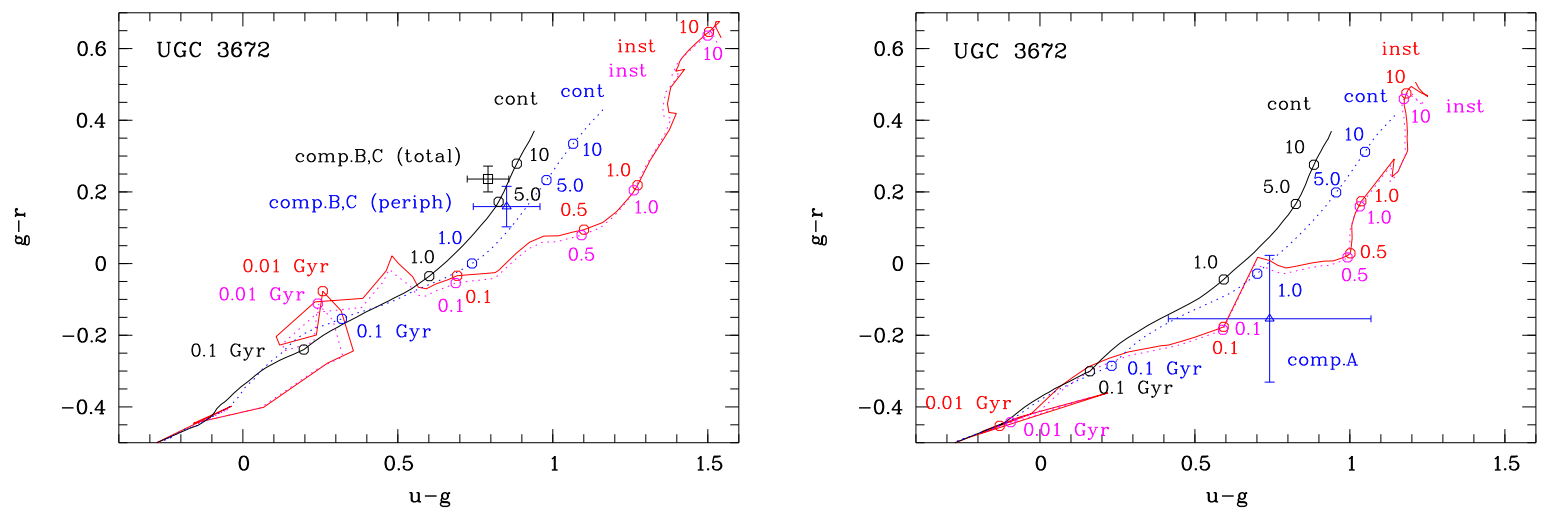

Figure 8. Extinction-corrected colours $g-r$ vs $u-g$ with their $\pm 1 \sigma$ uncertainties as measured on the SDSS DR12 images. Left panel: blue triangle - for outer parts of combined components B and C, black square - colours of B and C integrated light. Right panel: for the total light of component A. They are overlaid on the PEGASE2 package evolutionary tracks from Fioc \& Rocca-Volmerange (1999) with tickmarks in Gyr. Both tracks for continuous SF law with constant SFR (cont) and the instantaneous starburst (inst) are shown as extreme options. See more details in the text. For components B and C the metallicity of tracks is $z=0.004$, close to that of HII regions with $12+\log (\mathrm{O} / \mathrm{H}) \sim 8.0$. For component A we adopt the metallicity of tracks $z=0.0004$, corresponding to its tentative gas metallicity of $12+\log (\mathrm{O} / \mathrm{H})=7.0$ from Pustilnik, Perepelitsyna \& Kniazev (2016, MNRAS, submitted).

been an issue of interest. It has been known for decades that merger of disk galaxies could result in remnants that resemble ellipticals (e.g. Toomre \& Toomre 1972; Hernquist 1992; Barnes \& Hernquist 1996). However, in hierarchical galaxy formation models where mergers are expected to be frequent in the past, a more crucial question is as to whether disk galaxies can survive mergers.

While early simulations indicated that mergers result in the destruction of disks, it was later shown that for gas-rich disks (the so called "wet" mergers) even major mergers could result in disk-like morphology (Springel \& Hernquist 2005; Robertson et al. 2006; Hopkins et al. 2009). Loss of angular momentum of the gas which falls to the centre of the galaxy is what causes gas poor disks to get completely destroyed. Hopkins et al. (2009) argue that the angular momentum of the gas is lost by tidal torquing between the offset gas and stellar disks that are produced in the early stages of the interaction. For very gas-rich galaxies this tidal torquing is of less importance, and most of the gas retains its high angular momentum and resettles into a disk at the end of the merger. Bekki (2008) also presents the results of numerical simulations of mergers of extremely gas-rich dwarfs where some gas is driven inwards to fuel a central star burst, while the rest reforms into a disk. The physical mechanism which underlies this is however not discussed.

More recent simulations however show that not all "wet" mergers result in the re-formation of a disk. Bournaud et al. (2011) find that the effect of the gas richness on the merger remnant depends critically on whether the disk is dominated by clumpy turbulent gas. For disks dominated by clumpy turbulent gas, mergers do not produce long kinematically coherent tidal tails nor does the gas reform into a disk. They argue that since $z \sim 1-2$ galaxies appear to be better described as clumpy turbulent disks, it is unlikely that mergers of these galaxies could produce a remnant that is like the disk galaxies observed today. The morphology and kinematics of UGC 3672 indicates that this is not the situation we are dealing with here.

The final issue that we would like to draw the readers attention to is the spatial distribution of the galaxies in the system. As argued above, UGC 3672 can be best understood as an interacting triplet of galaxies, with the galaxies in the triplet having a more or less linear alignment. This is similar to the case of the triplet found by Beygu et al. (2013) in their Void Galaxy Survey, and which they suggest reflects the underlying filamentary nature of the distribution of galaxies in voids. The kinematical continuity that we find across the UGC 3672 system is also similar to that noted in the study of the NGC 672 and NGC 784 groups by Zitrin \& Brosch (2008). These authors suggest that the kinematical alignment arises because the galaxy in the groups like along a filament of the large scale structure.

The galaxy triplet discovered earlier near the centre of the Lynx-Cancer void (Chengalur \& Pustilnik 2013) also has a more or less linear geometry, although in this triplet there is no bridge of emission connecting the third galaxy with the main pair, and although there is velocity continuity across the main pair, it does not extend to the third galaxy. The linear arrangement and velocity continuity of the galaxies in the UGC 3672 system, along with the fact that the diffuse gas appears to be settling into a rotating disk, indicate that wet mergers with flow along filaments is a possible way to produce disk-like systems.

\section{SUMMARY AND CONCLUSIONS}

We present multi-resolution $\mathrm{HI}$ images and optical photometry data of the galaxy UGC 3672, which is located near the centre of the nearby Lynx-Cancer void. From the data analysis and their discussion in the broader context, we draw the following conclusions.

(i) At low spatial resolution, the Hi distribution appears to be in a clumpy disk with fairly regular dynamics. At this resolution, the Hi distribution and kinematics is consistent with what is typical of dwarf irregular galaxies.

(ii) At high spatial resolutions however, the Hi distribution is highly unusual, consisting of a secondary peak, which 
is identified with a separate, much fainter dwarf irregular galaxy, as well as several filamentary structures.

(iii) This faint LSB dwarf UGC 3672A appears extremely gas-rich, with the ratio $M(\mathrm{HI}) / L_{\mathrm{B}}=17.1$. Besides, its integrated colours $u-g$ and $g-r$, when compared with the PEGASE2 package evolutionary tracks indicate that the bulk of the stars in this system have formed recently. This, along with its extremely low metalicity $\left(\mathrm{O} / \mathrm{H}\right.$ of $\left.\sim(\mathrm{O} / \mathrm{H})_{\odot} / 50\right)$ means that UGC $3672 \mathrm{~A}$ has all the properties expected of unevolved galaxies. Along with two other extremely gas-rich and blue LSBDs, from another triplet J0723+36 near the void centre, residing at only $\sim 2 \mathrm{Mpc}$ from UGC 3672 system, this finding provides an additional evidence for the unevolved nature of small dwarfs near the void centres.

(iv) Arguments are presented that UGC 3672 system is best understood as representing the merger of what was originally a triplet of very gas-rich dwarf galaxies. We suggest that the location of this highly unusual system is not a coincidence but is related to the "cosmic microscope and time machine" effects associated with voids.

(v) Our observations indicate that wet mergers of galaxies flowing along a filament is a possible pathway for the production of disk like systems.

\section{ACKNOWLEDGEMENTS}

We thank the staff of the GMRT who made these observations possible. The GMRT is run by the National Centre for Radio Astrophysics of the Tata Institute of Fundamental Research. The work of SAP and ESE was supported by the RSCF grant No. 14-12-00965. We acknowledge the use of the SDSS DR12 database. Funding for the Sloan Digital Sky Survey (SDSS) has been provided by the Alfred P. Sloan Foundation, the Participating Institutions, the National Aeronautics and Space Administration, the National Science Foundation, the U.S. Department of Energy, the Japanese Monbukagakusho, and the Max Planck Society. The SDSS Web site is http://www.sdss.org/ The SDSS is managed by the Astrophysical Research Consortium (ARC) for the Participating Institutions. This research has made use of the NASA/IPAC Extragalactic Database (NED) which is operated by the Jet Propulsion Laboratory, California Institute of Technology, under contract with the National Aeronautics and Space Administration. We are grateful to the anonymous referee whose comments helped improve this paper.

\section{REFERENCES}

Alam S., Albareti F. D., Allende Prieto C., et al. ., 2015, ApJS, 219, 27

Aragon-Calvo M. A., Szalay A. S., 2013, MNRAS, 428, 3409

Barnes J. E., Hernquist L., 1996, ApJ, 471, 115

Begum A., Chengalur J. N., Karachentsev I. D., Sharina

M. E., Kaisin S. S., 2008, MNRAS, 386, 1667

Bekki K., 2008, MNRAS, 388, L10

Bekki K., 2015a, MNRAS, 454, L41

Bekki K., 2015b, ApJL, 812, L14
Berg D. A., Skillman E. D., Marble A. R., van Zee L., Engelbracht C. W., Lee J., Kennicutt R. C., Calzetti D., Dale D., Johnson B., 2012, ApJ, 754, 1

Beygu B., Kreckel K., van de Weygaert R., van der Hulst J. M., van Gorkom J. H., 2013, AJ, 145, 120

Biswas R., Alizadeh E., Wandelt B. D., 2010, Ph.Rv.(D), 82, 023002

Bournaud F., Chapon D., Teyssier R., Powell L. C., Elmegreen B. G., Elmegreen D. M., Duc P.-A., Contini T., Epinat B., Shapiro K. L., 2011, ApJ, 730, 4

Cai Y.-C., Padilla N., Li B., 2015, MNRAS, 451, 1036

Cannon J. M., Johnson M., McQuinn K. B. W., Alfvin E. D., Bailin J., Ford H. A., Girardi L., Hirschauer A. S., et. al. 2014, ApJL, 787, L1

Chengalur J. N., 2013, Technical Report NCRA/COM/001, FLAGCAL: a flagging and calibration pipeline for GMRT DATA. NCRA-TIFR

Chengalur J. N., Pustilnik S. A., 2013, MNRAS, 428, 1579

Chengalur J. N., Pustilnik S. A., Makarov D. I., Perepelitsyna Y. A., Safonova E. S., Karachentsev I. D., 2015, MNRAS, 448, 1634

Dubinski J., da Costa L. N., Goldwirth D. S., Lecar M., Piran T., 1993, ApJ, 410, 458

Ekta Chengalur J. N., Pustilnik S. A., 2008, MNRAS, 391, 881

Ekta B., Chengalur J. N., 2010, MNRAS, 406, 1238

Fioc M., Rocca-Volmerange B., 1999, p. arXiv/9912.179

Goldberg D. M., Vogeley M. S., 2004, ApJ, 605, 1

Gottlöber S., Łokas E. L., Klypin A., Hoffman Y., 2003, MNRAS, 344, 715

Grogin N. A., Geller M. J., 1999, AJ, 118, 2561

Guseva N. G., Izotov Y. I., Fricke K. J., Henkel C., 2015, A\&A, 579, A11

Hernquist L., 1992, ApJ, 400, 460

Hibbard J. E., Bianchi L., Thilker D. A., Rich R. M., Schiminovich D., Xu C. K., Neff S. G., Seibert M. e. a., 2005, ApJL, 619, L87

Hirschauer A. S., Salzer J. J., Skillman E. D., Berg D., McQuinn K. B. W., Cannon J. M., Gordon A. J. R., Haynes M. P., Giovanelli R., Adams E. A. K., Janowiecki S., Rhode K. L., Pogge R. W., Croxall K. V., Aver E., 2016, arxiv:1603.03798

Hoeft M., Yepes G., Gottlöber S., Springel V., 2006, MNRAS, 371, 401

Hopkins P. F., Cox T. J., Younger J. D., Hernquist L., 2009, ApJ, 691, 1168

Hoyle F., Rojas R. R., Vogeley M. S., Brinkmann J., 2005, ApJ, 620, 618

Hoyle F., Vogeley M. S., Pan D., 2012, MNRAS, 426, 3041

Hunter D. A., Elmegreen B. G., 2004, AJ, 128, 2170

Izotov Y. I., Thuan T. X., 2007, ApJ, 665, 1115

Izotov Y. I., Thuan T. X., Guseva N. G., 2012, A\&A, 546, A122

Jõeveer M., Einasto J., Tago E., 1978, MNRAS, 185, 357

Karachentsev I. D., Makarov D. I., Karachentseva V. E., Melnyk O. V., 2011, Astrophysical Bulletin, 66, 1

Kirshner R. P., Oemler Jr. A., Schechter P. L., Shectman S. A., 1981, ApJL, 248, L57

Kreckel K., Peebles P. J. E., van Gorkom J. H., van de Weygaert R., van der Hulst J. M., 2011, AJ, 141, 204

Liu C.-X., Pan D. C., Hao L., Hoyle F., Constantin A., Vogeley M. S., 2015, ApJ, 810, 165 
Moiseev A. V., 2014, Astrophysical Bulletin, 69, 1

Moorman C. M., Moreno J., White A., Vogeley M. S., Hoyle

F., Giovanelli R., Haynes M. P., 2016, arxiv:1601.04092

Moorman C. M., Vogeley M. S., Hoyle F., Pan D. C., Haynes M. P., Giovanelli R., 2014, MNRAS, 444, 3559

Neff S. G., Thilker D. A., Seibert M., Gil de Paz A., Bianchi L., Schiminovich D., Martin D. C., Madore B. F. e. a., 2005, ApJL, 619, L91

Padmanabhan N., Schlegel D. J., Finkbeiner D. P., et al. ., 2008, ApJ, 674, 1217

Park D., Lee J., 2007, Physical Review Letters, 98, 081301

Peebles P. J. E., 2001, ApJ, 557, 495

Perepelitsyna Y. A., Pustilnik S., Kniazev A., 2014, Astrophysical Bulletin, 69, 247

Prasad J., Chengalur J., 2012, Experimental Astronomy, 33, 157

Pustilnik S. A., Kniazev A. Y., Pramskij A. G., 2005, A\&A, 443, 91

Pustilnik S. A., Martin J.-M., 2016, arXiv:1608.08284

Pustilnik S. A., Martin J.-M., Tepliakova A. L., Kniazev A. Y., 2011, MNRAS, 417, 1335

Pustilnik S. A., Perepelitsyna Y. A., Kniazev A. Y., 2016, MNRAS, 463, 670

Pustilnik S. A., Tepliakova A. L., 2011, MNRAS, 415, 1188

Pustilnik S. A., Tepliakova A. L., Kniazev A. Y., 2011, Astrophysical Bulletin, 66, 255

Pustilnik S. A., Tepliakova A. L., Kniazev A. Y., Martin J.-M., Burenkov A. N., 2010, MNRAS, 401, 333

Rieder S., van de Weygaert R., Cautun M., Beygu B., Portegies Zwart S., 2013, MNRAS, 435, 222

Robertson B., Bullock J. S., Cox T. J., Di Matteo T., Hernquist L., Springel V., Yoshida N., 2006, ApJ, 645, 986

Rojas R. R., Vogeley M. S., Hoyle F., Brinkmann J., 2004, ApJ, 617, 50

Rojas R. R., Vogeley M. S., Hoyle F., Brinkmann J., 2005, ApJ, 624, 571

Sahni V., Sathyaprakah B. S., Shandarin S. F., 1994, ApJ, 431, 20

Sanchez Almeida J., Elmegreen B. G., Muñoz-Tuñón C., Elmegreen D., 2014, The Astronomy and Astrophysics Review, 22, 1

Sanchez Almeida J., Elmegreen B. G., Muñoz-Tuñón C., Elmegreen D. e. a., 2015, ApJ, 810, 1

Schlafly E. F., Finkbeiner D. P., 2011, ApJ, 737, 1

Sheth R. K., van de Weygaert R., 2004, MNRAS, 350, 517

Springel V., Hernquist L., 2005, ApJL, 622, L9

Springob C. M., Haynes M. P., Giovanelli R., Kent B. R., 2005, ApJS, 160, 149

Sutter P. M., Lavaux G., Wandelt B. D., Weinberg D. H., 2012, ApJ, 761, 187

Thuan T., Lecavelier des Etangs A., Izotov Y. I., 2005, ApJ, 621, 269

Toomre A., Toomre J., 1972, ApJ, 178, 623

Tully R. B., Shaya E. J., Karachentsev I., Courtois H. M., Kocevski D. D., Rizzi L., Peel A., 2008, ApJ, 676, 184 van Zee L., 2000, AJ, 119, 2757

van Zee L., Haynes M. P., Salzer J. J., 1997, AJ, 114, 2479

van Zee L., Maddalena R. J., Haynes M. P., Hogg D. E., Roberts M. S., 1997, AJ, 113, 1638

Yun M. S., Ho P. T. P., Lo K. Y., 1994, Nature, 372, 530

Zitrin A., Brosch N., 2008, MNRAS, 390, 408
Zivick P., Sutter P. M., Wandelt B. D., Li B., Lam T. Y., 2015, MNRAS, 451, 4215 